\section{Comparison of muscle activation of 3 different hip belt squat techniques}

\author{
Colleen N Gulick ${ }^{1 *}$ and Dawn T Gulick ${ }^{2}$ \\ ${ }^{1}$ Podium Sports LLC, Founder \& CEO, Spring City, PA, USA \\ ${ }^{2}$ Widener University, Institute for Physical Therapy Education, Chester, PA, USA
}

\section{Abstract}

The purpose of this study was to differentiate between muscular activity of three different types of belt squats (SquatMax-MD, Pit Shark and Monster Rhino) and the muscle activation of the rectus femoris, vastus medialis oblique, gluteus maximus, and gluteus medius. Fourteen healthy, male athletes, over the age of 18 years, performed 2 sets of 5 repetitions on each of the three belt squat machines with a weight equivalent to each participant's body weight. Athletes were given at least 2 minutes of rest between each set and condition. Electromyographic data were collected from four muscles: rectus femoris, vastus medialis oblique, gluteus maximus, and gluteus medius muscles. ANOVA revealed the SquatMax-MD belt squat resulted in the highest muscle activation in every muscle, with significantly higher activity in the rectus femoris, vastus medialis oblique, and gluteus medius muscles. The Monster Rhino belt squat produced the second highest muscle activation with the Pit Shark belt squat creating the lowest muscle activation. In totality, the SquatMax-MD produced $38.7 \%$ greater muscle activation than the Monster Rhino and $12.2 \%$ greater activation than the Pit Shark. The belt squat can be an advantageous exercise because it can effectively load the lower body while de-loading the spine and upper body. The difference in activation between the SquatMax-MD and other belt squats may be due, in part, to the design of the machines. The additional activation produced by the SquatMax-MD belt squat may be useful for individuals seeking hypertrophy, strength, or a reduction in injury risk.

\section{More Information}

*Address for Correspondence: Colleen $\mathrm{N}$ Gulick, PhD (ABD), MS, BS (BioE), CSCS, 64 Bethel Church Road, Spring City, PA, USA, Email: cgulick11@gmail.com

Submitted: 29 August 2020

Approved: 14 September 2020

Published: 15 September 2020

How to cite this article: Gulick CN, Gulick DT. Comparison of muscle activation of 3 different hip belt squat techniques. J Nov Physiother Rehabil. 2020; 4: 034-039.

DOI: 10.29328/journal.jnpr.1001035

ORCiD: orcid.org/0000-0002-2122-5466

Copyright: () 2020 Gulick CN, et al. This is an open access article distributed under the Creative Commons Attribution License, which permits unrestricted use, distribution, and reproduction in any medium, provided the original work is properly cited.

Keywords: Squat; Belt squat; Muscle recruitment; EMG

Check for updates

OPEN ACCESS

\section{Introduction}

The quest for new methods to improve strength and athletic performance is perpetual. The squat exercise is frequently employed by both healthy and rehabilitating athletes. This closed-kinetic chain task is popular for reducing tibial-femoral shear forces in anterior cruciate ligament (ACL) rehabilitation [1], patella-femoral tracking dysfunctions (PFTD), and total knee replacement therapy [2]. However, it is not always applicable or advantageous to use a traditional back squat. Individuals with various co-morbidities of the shoulder or spine may experience limitations in performing this exercise. People with a history of shoulder impingement, labral injuries, or thoracic outlet may not be able to position the bar correctly behind the shoulders in order to perform a traditional back squat [3]. In addition, individuals with a history of lumbosacral dysfunction may have difficulty using a traditional back squat to challenge the lower extremities with a load that does not surpass the tolerance of the spine [3]. Therefore, an alternative exercise, such as the belt squat, can prioritize the load to the lower body musculature and be an effective substitute for the traditional back squat.

All belt squats utilize a belt to place the load on the hips, effectively circumventing the need to support the weight with the upper extremities. However, the way in which the belt attaches to the weight differs between belt squat designs. The three most used hip belt squat designs currently on the market are: SquatMax-MD (OverAchieve Sports and Speed, King of Prussia, PA), Pit Shark (Beachside Fitness Equipment, New Smyrna Beach, FL), Monster Rhino (Rogue Fitness, Columbus $\mathrm{OH}$ ). The SquatMax-MD hip belt uses a free-weight-on-guiderod design to keep the weight centered directlfy under the lifter (Figure 1). The belt is attached to one of three hook-up points, allowing the athlete to choose to affix the belt to a rear, central, or forward point of attachment. The Pit Shark uses a lever belt squat design (Figure 2). A hook is used to attach the belt to one of four hook-up points, allowing the user to choose the position of attachment in the sagittal plane. In this way, the weight moves along a fixed track throughout a lifter's range of motion. In the Pit Shark design, the weight is added laterally, similar to a barbell, in front of the lever arm. The Monster Rhino belt squat (stand-alone version) uses a cable attachment in which the athlete attaches to a single carabiner at the end of a cable extruding from the middle of the platform (Figure 3). Weight is loaded in front of the platform, horizontally. Similar to the Pit Shark Design, the weight moves along a fixed track. 


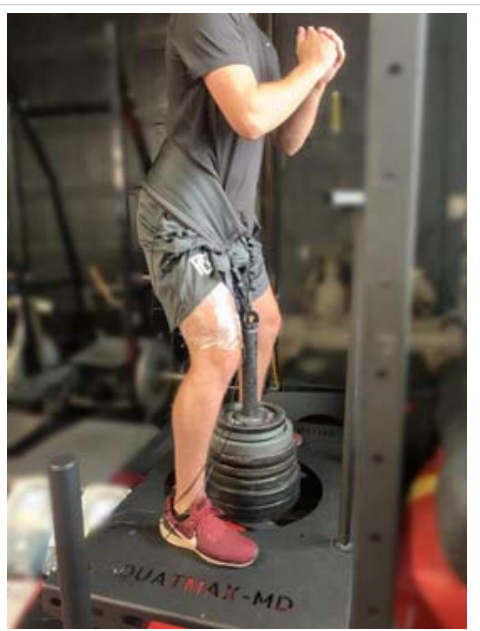

Figure 1: SquatMax-MD belt squat.

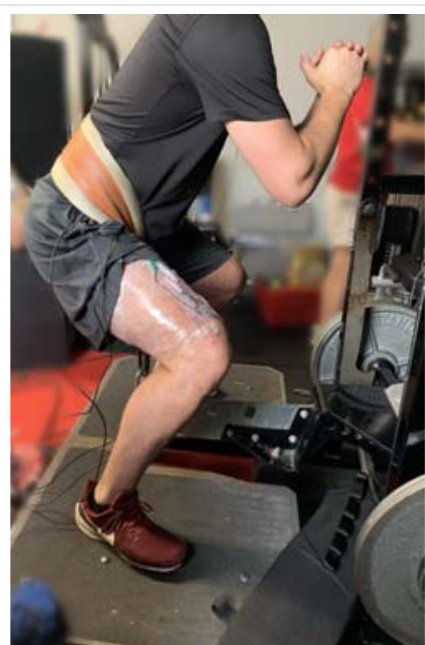

Figure 2: Pit Shark belt squat.

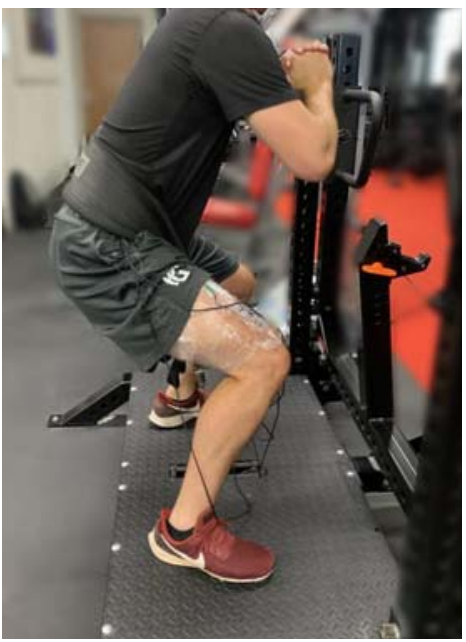

Figure 3: Monster Rhino belt squat.

Upon standing, the trolley will rise and disengage from the Rhino Horn, allowing the athlete to perform the desired squat repetitions.

In the published research to date, the SquatMax-MD freeweight belt squat design has been shown to have similar lower body muscle activation to the barbell back squat (quadriceps, biceps femoris, hip adductors, hip abductors, gluteus maximus, gastrocnemius) [4]. Evans, et al. [5] and Joseph, et al. [6] utilized two different lever arm belt squat designs, Pit Shark and Wenning Strength belt squat machines respectively, and found the muscle activation of the belt squat to be similar to back squats in the quadriceps, hamstring, and plantar flexor muscles. However, both studies found the lever arm belt squat designs to have significantly lower gluteus maximus muscle activation when compared to the back squat. In addition, Joseph, et al. [6] found the lumbar erectors, gluteus medius, rectus abdominus and external oblique muscles to have significantly lower muscle activation when utilizing the Wenning Strength belt squat. Previous researchers [6] hypothesized that the difference in findings between studies may be related to the differences in belt squat designs, with weight anchored to a fixed point versus free-weight-on-guiderod. Therefore, the purpose of this study was to expand upon the previous research and use electromyography (EMG) differentiate between muscular activity of three different types of belt squats (SquatMax-MD, Pit Shark and Monster Rhino) and the muscle activation of the rectus femoris (RF), vastus medalis oblique (VMO), gluteus maximus (Gmax), and gluteus medius (Gmed).

\section{Methods}

\section{Participants}

Fourteen healthy, male athletes, over the age of 18 years, were recruited. Each participant was a competitive athlete who was competent with the squat motion on all three belt squat variations. All anthropometric data were recorded (Table 1). None of the volunteers had a current musculoskeletal injury or any injury/surgery in the past 12 months. All participants signed a consent form approved by the university institutional review board for the protection of human subjects. The study conformed with the Code of Ethics of the World Medical Association (Declaration of Helsinki).

\section{Equipment}

Two Chattanooga surface EMG apparatuses (DJO Global, Vista, CA) were used to assess muscle activity. A SquatMaxMD hip belt squat platform with rear point of attachment (King of Prussia, PA), Pit Shark belt squat (Beachside Fitness Equipment, New Smyrna Beach, FL), and Monster Rhino belt squat (Columbus, $\mathrm{OH}$ ) were used to compare lifting techniques. Standard lifting plates of various increments $(2.5,5,10,15$, $20 \mathrm{~kg}$ ) were used to provide resistance in the amount of the participant's body weight to each lifting technique.

\begin{tabular}{|c|c|c|}
\hline Table 1: Participant Descriptive Characteristics $(n=14)$. \\
\hline Age $(\mathrm{y})$ & Mean & SD \\
\hline Height $(\mathrm{cm})$ & 21.29 & 2.92 \\
\hline Weight $(\mathrm{kg})$ & 182.14 & 5.92 \\
\hline Squat weight used $(\mathrm{kg})$ & 88.65 & 13.98 \\
\hline
\end{tabular}




\section{Procedures}

After informed consent was obtained, anthropometric data were collected (age, height, weight). Each athlete was then prepped for EMG electrode placement in a private area. Anatomic locations were identified for each muscle. Upon contraction each muscle was palpated, the skin was cleaned with alcohol wipes, and the hair trimmed and/or adhesive sprayed in the area to assure firm electrode adherence. The EMG electrodes were placed on the muscle belly, parallel to the line of action on the right side of the body on the following muscles: RF, VMO, Gmax, and Gmed based on the instructions by Florimond, [7] and Gullet, et al. [8]. Reference images of the electrode placement can be found online in the Florimond, [7] manuscript on pages 18 and 19. After secure placement, the electrodes and their corresponding wires were wrapped onto the muscle. Electrodes stayed affixed to the participant's skin for all three conditions. Surface electrodes were chosen, hence the system was non-invasive and painless [9].

The athletes were randomly assigned to the order of data collection (Squat-Max-MD, Pit Shark, Monster Rhino) with all data collected in one session for consistency of the EMG electrode placement. The sequence for each device was as follows:

The athlete's body weight (rounded down to the nearest $2.5 \mathrm{~kg}$ increment) was racked for each athlete (Table 1);

The athlete donned the specific belt for the device and placed his feet shoulder width apart (on either side of the cylinder, lever or cable depending upon the device) to standardize positioning $[1,10]$ and placed arms in front of the chest;

The athletes were instructed to perform the squats in a controlled manner without holding on with their hands;

The athletes were instructed to squat down to a level at which the knees are at 90 degrees of flexion and the thighs are parallel to the floor. Squat depth was visually observed by the primary investigator with feedback to the athlete to maintain consistency across repetitions and conditions;

The athletes performed 2 sets of 5 continuous repetitions of each squat technique with a minimum of 2 minutes of rest between each set to allow for adequate muscle recovery. The first set of each condition served as a warm up set and EMG data was collected for the 5 repetitions of the second set of each squat technique;

After the second set, the athletes were given at least 2 minutes of rest then moved to the second and third squat technique for data collection.

\section{Statistics}

All data analyses were performed using Stata V.16 software (StataCorp LP, College Station, TX) with an alpha level of $p=0.05$. Normality of the data was assessed using a ShapiroWilk test. A repeated measures ANOVA was performed to investigate the muscle activation differences between squat techniques in each of the four muscles measured (RF, VMO, Gmax, Gmed). Variance was addressed via the Maulchy's test of sphericity. Results that failed to satisfy the assumption of sphericity were corrected using the Greenhouse-Geisser correction.

\section{Results}

The age, stature, body weight, and squat weight used by each of the participants is described in table 1. Root Mean Square (RMS) is the technique used for rectifying the raw signal to convert it to an amplitude envelope and make it easier to view. The mean, standard error, and confidence interval of EMG muscle activity for each squat technique is displayed in table 2 .

The repeated measures ANOVA showed the muscle activation in each of the RF, VMO, and Gmed muscles were significantly different between squat techniques (Table 3 ). A graph for each muscle group by device is displayed in figures 4,5 with post-hoc test results presented in table 4 . The SquatMax-MD resulted in the highest muscular activation for every muscle. When compared to the Pit Shark, the SquatMaxMD had significantly greater activation in the RF, VMO, and Gmed muscles. The SquatMax-MD produced significantly greater muscle activation than the Monster Rhino in the VMO and Gmed muscles. The Monster Rhino had the second highest muscle activation for every muscle. When compared to the Pit Shark, the Monster Rhino had significantly greater activation in the RF and VMO muscles. Figures 1-3 shows the same participant on the SquatMax-MD, Pit Shark, and Monster Rhino machines, respectively.

Table 2: Mean, standard error, and confidence intervals of Electromyographic (EMG) activity for each belt squat technique.

\begin{tabular}{|c|c|c|c|c|c|}
\hline Muscle & Squat Type & Mean (uV) & SE & $\begin{array}{c}95 \% \text { Cl } \\
\text { Lower Limit }\end{array}$ & $\begin{array}{c}95 \% \text { Cl } \\
\text { Upper Limit }\end{array}$ \\
\hline & & & & & \\
\hline RF & SquatMax-MD & 608.67 & 72.23 & 461.63 & 755.7 \\
\hline & Pit Shark & 379 & 74.04 & 228.37 & 529.63 \\
\hline \multirow{2}{*}{ VMO } & Monster Rhino & 579.42 & 69.36 & 438.3 & 720.54 \\
\hline & SquatMax-MD & 634.92 & 68.72 & 495.1 & 774.74 \\
\hline \multirow{2}{*}{ Gmax } & Pit Shark & 346 & 32.15 & 280.59 & 411.41 \\
\hline & Monster Rhino & 531.67 & 44.45 & 441.23 & 622.1 \\
\hline & SquatMax-MD & 161.25 & 16.6 & 127.49 & 195.01 \\
\hline \multirow{2}{*}{ Gmed } & Pit Shark & 143 & 14.34 & 113.82 & 172.18 \\
\hline & Monster Rhino & 150 & 15.48 & 118.5 & 181.5 \\
\hline & SquatMax-MD & 166.67 & 14.88 & 136.4 & 196.94 \\
\hline & Pit Shark & 96 & 10.02 & 75.61 & 116.39 \\
\hline & Monster Rhino & 119.17 & 14.11 & 90.45 & 147.88 \\
\hline
\end{tabular}

\begin{tabular}{|c|c|c|c|}
\hline \multicolumn{3}{|c|}{ Table 3: Analysis of variance (ANOVA) test results } \\
\hline Muscle & F value & DF & $\boldsymbol{p}$ - value \\
\hline RF & 8.63 & 2 & 0.0014 \\
\hline VMO & 26.25 & 2 & $<0.001$ \\
\hline Gmax & 0.31 & 2 & 0.734 \\
\hline Gmed & 5.19 & 2 & 0.0143 \\
\hline
\end{tabular}


Table 4: Analysis of Variance (ANOVA) post-hoc comparisons by squat type for each statistically significant muscle

\begin{tabular}{|c|c|c|c|c|c|c|}
\hline Muscle & Squat Type & Contrast & Std Err & $P>t$ & $95 \%$ Cl Low & $95 \%$ Cl High \\
\hline \multirow[t]{3}{*}{ RF } & Squatmax-MD vs Pit Shark & -211.36 & 56.24 & 0.001 & -327.18 & -95.54 \\
\hline & Squatmax-MD vs Monster Rhino & -16.06 & 57.84 & 0.78 & -135.18 & 103.05 \\
\hline & Monster Rhino vs Pit Shark & 195.29 & 57.84 & 0.002 & 76.18 & 314.41 \\
\hline \multirow[t]{3}{*}{ VMO } & Squatmax-MD vs Pit Shark & -247.07 & 34.18 & $<0.001$ & -317.33 & -176.81 \\
\hline & Squatmax-MD vs Monster Rhino & -108.86 & 34.18 & 0.004 & -179.12 & -38.6 \\
\hline & Monster Rhino vs Pit Shark & 138.21 & 34.18 & $<0.001$ & 67.96 & 208.47 \\
\hline \multirow[t]{3}{*}{ Gmed } & Squatmax-MD vs Pit Shark & -49.2 & 17.39 & 0.01 & -85.27 & -13.13 \\
\hline & Squatmax-MD vs Monster Rhino & -43.85 & 16.32 & 0.013 & -77.68 & -10.01 \\
\hline & Monster Rhino vs Pit Shark & 5.35 & 17.39 & 0.761 & -30.72 & 41.42 \\
\hline
\end{tabular}

*Gmax does not appear in the post-hoc Table since the repeated measures ANOVA did not reveal a significant difference between squat types for this muscle



Figure 4: Quadricep Electromyographic (EMG) Activity.

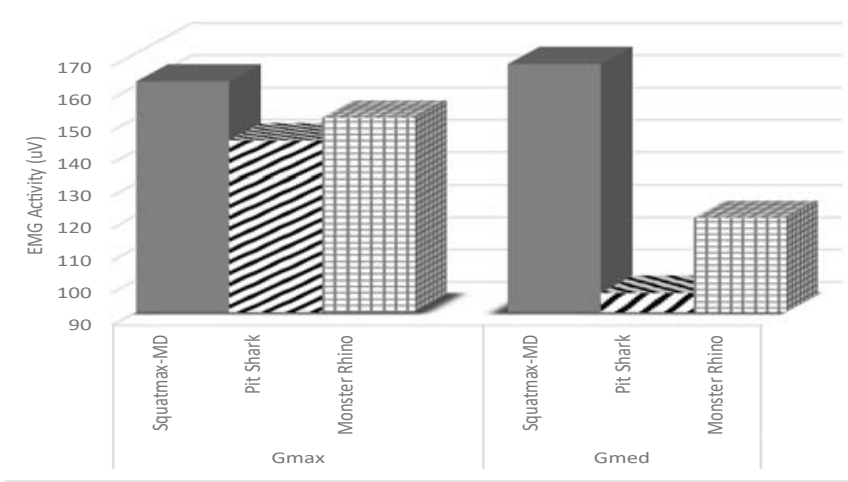

Figure 5: Gluteal Electromyographic (EMG) Activity.

\section{Discussion}

This study was the first to quantify the differences in muscle activation between three different types of belt squats. The findings revealed a significant difference between squat types in the muscle activation of the RF, VMO, and Gmed muscles. The SquatMax-MD resulted in the highest muscle activation in every muscle. The Monster Rhino belt squat produced the second highest muscle activation with the Pit Shark belt squat creating the lowest muscle activation. In totality, the SquatMax-MD produced $38.7 \%$ greater muscle activation than the Monster Rhino and $12.2 \%$ greater activation than the Pit Shark. A previous study [4] found the muscular activation of the SquatMax-MD to be comparable to a barbell back squat. Given that the Monster Rhino and Pit Shark elicited significantly less muscular activation than the SquatMax-MD in this study, one can reasonable conclude both the Monster
Rhino and Pit Shark require significantly less activation than a barbell back squat.

Possible reasons for the significantly higher Gmed and slightly increased Gmax activity in the SquatMax-MD may include the point of attachment. A previous study by Evans, et al. [5] utilized the Pit Shark and appears (based on figure 2 Evans, et al. [5]) to have had their participants stand at the rear edge of the platform and attach to the second most posterior point on the lever arm. The current study had all participants attach to the most posterior point on the lever arm. It is likely that a different angle of pull altered the kinematics of the athlete's squat, thereby influencing muscle recruitment. The current study, utilizing the most posterior point of attachment for both the SquatMax-MD and Pit Shark should, biomechanically, have resulted in maximal gluteal activation. Any other placement would likely pull the athlete forward, increasing forward translation of the knee, and subsequently decreasing gluteal activation.

While the weight lifted was standardized for all three conditions, the SquatMax-MD resulted in increased activation for all four muscles. One possible reason for this uniformly higher muscle activation may be due to differences in belt squat designs. Previous studies have shown that exercises in which the weight moves along a fixed track have significantly less muscle activation than free weight exercises [11-13]. Schwanbeck, et al. [12] investigated the EMG activity of a free weight squat as compared to a Smith machine and found $43 \%$ higher muscle activation when using the free weight squat. It was surmised that the closed chain task increases knee compressive forces and stimulated a joint co-contraction. Likewise, Escamilla, et al. [11] revealed that the muscular activity was significantly higher when participants performed a squat (closed kinetic chain) as opposed to a leg press (open kinetic chain), regardless of using a high or low foot position. Both the Pit Shark and the Monster Rhino are designs in which the weight moves along a fixed track (Video 1, Video 2 respectively). This can reduce the need for the recruitment of musculature stabilizers since the track of movement is defined. Whereas, the free-weight-on-guide-rod design of the SquatMax-MD requires the athlete to control the path of the weight, potentially increasing the need to contract stabilizing muscles, and may be one of the reasons for the increased muscular activation (Video 3). 
Numerous research studies have demonstrated the importance of hip abduction and external rotation strength in improving landing technique, preventing ACL and PFTD [14-19]. Khayambashi, et al. [20] identified the importance of hip external rotation and abduction strength in preventing ACL injuries in both male and female athletes. Their research resulted in clinical cutoffs, defining athletes as high risk for a noncontact ACL injury if their hip external rotation strength is less than or equal to $20.3 \%$ of body weight or hip abduction strength less than or equal to $35.4 \%$ of body weight [20]. Thus, in order to mitigate the risk of ACL injury, athletes should be advised to strengthen their hip external rotators and abductors. Both the Gmax and Gmed muscles perform hip external rotation and abduction, respectively. Since the SquatMax-MD demonstrated significantly higher Gmed activity than the Pit Shark and Monster Rhino belt squats, the SquatMax-MD may be a useful tool for individuals seeking to strengthen their hip external rotators and abductors, thereby decreasing their risk of ACL injury and improving patella tracking.

Although it is not the intention of this study to seek subjective feedback from the athletes, many provided comments about the various devices. Anecdotally, the participants of the study appeared to prefer the SquatMax-MD and Pit Shark squats as opposed to the Monster Rhino. They reported difficulty setting up the Monster Rhino belt squat due to the lower point of attachment than the other two belt squat designs. The Monster Rhino forced them to get into a deeper squat than the other two designs in order to begin the lift. In addition, athletes made comments while using the Monster Rhino that they felt as if they were being pulled forward, their heels were coming off the platform, and one athlete indicated that his toes started to cramp when attempting to counteract the perceived lumbar lordosis. These comments may be due to the forward angle of pull created by the Monster Rhino attachment, as evidenced by video 2 . Athletes expressed that the lift was more challenging on the SquatMax-MD device, even though the weights were standardized between the three conditions. These anecdotes are substantiated by the increased EMG activity when using the SquatMax-MD belt squat. While we did not perform a biomechanical analysis, the Pit Shark appeared to alter the kinematics of the taller athletes, with the belt length and fixed point of attachment limiting their posterior excursion. Shorter athletes did not appear to have this limitation and were able to maintain their preferred squat form throughout the lift.

\section{Strengths and limitations}

Given that previous studies have already compared the muscular activation of the SquatMax-MD [4] and Pit Shark [5] belt squats to a parallel back squat, the authors chose to focus this study on comparing three different types of belt squat. The muscles selected in the current study were based on a thorough review of the literature. The hamstrings were not assessed. It is possible that hamstring activity is different between these belt squat designs. However, the squat exercise is not known for high hamstring activity [21], with prior studies reporting that the squat only yielded $27 \%$ of maximal voluntary isometric hamstring activity [15,22]. Thus, we selected to focus on the quadriceps and gluteal muscles for the present study.

Previous studies have investigated the influence of foot position, squat depth, and stance width $[2,23,24]$ on muscular activation. In this study, all of these variables were consistent across all three conditions. Future studies conducting kinematic analyses of the knee joint in each squat may be helpful. Further discussion on the influence of these parameters is beyond of the scope of this study.

There are additional opportunities for future examination of these three devices. The SquatMax-MD device has three possible points of attachment, the Pit Shark has four possible points of attachment, and the Monster Rhino has a single point of attachment. Repeating this methodology on a variety of combinations of attachments could be a useful addition to the literature.

\section{Practical applications}

The belt squat can be an advantageous exercise because it can effectively load the lower body while de-loading the spine and upper body. When comparing three different types of belt squats, the SquatMax-MD produced significantly higher activation in the RF, VMO and Gmed musculature. The difference in activation between the SquatMax-MD and other belt squats may be due, in part, to the design of the machines. The free-weight-on-guide-rod design of the SquatMax-MD may require more stabilization, and thus require increased muscular activation. However, this muscular recruitment can be reduced when the belt squat design involves weight movement along a fixed track, such as the Pit Shark and Monster Rhino. Thus, the additional activation produced by the SquatMax-MD belt squat may be useful for individuals seeking to increase hypertrophy or strength. Furthermore, the increased Gmax and Gmed activation produced by the SquatMax-MD has the potential to facilitate hip external rotation and abduction strength, subsequently reducing an individual's risk of injury.

\section{Acknowledgement}

The authors claim no conflict of interest; the results of the current study do not constitute endorsement of the equipment.

\section{References}

1. Paoli A, Marcolin G, Petrone N. The effect of stance width on the electromyographical activity of either superficial thigh muscles during back squat with different bar loads. J Strength Cond Res. 2009; 23: 246-250.

PubMed: https://pubmed.ncbi.nlm.nih.gov/19130646/

2. Dionisio V, Almeida G, Duarte M, Hirata R. Kinematic, kinetic \& EMG 
patterns during downward squatting. J Electromyogr Kinesiol. 2008; 18: $134-143$.

PubMed: https://pubmed.ncbi.nlm.nih.gov/17029862/

3. Comfort P, Kasim P. Optimizing Squat Technique. Strength and Conditioning J. 2007; 29: 10-13.

4. Gulick D, Fagnani J, Gulick C. Comparison of muscle activation of hip belt squat and barbell back squat techniques. Isokinet Exerc Sci. 2015; 23: 101-108.

5. Evans T, McLester C, Howard J, McLester J, Calloway J. Camparison of Muscle Activation Between Back Squats and Belt Squats. J Strength Cond Res. 2019; 33: S52-S59.

PubMed: https://pubmed.ncbi.nlm.nih.gov/28595237/

6. Joseph L, Reilly J, Sweezey K, Waugh R, Carlson L, et al. Activity of Trunk and Lower Extremity Musculature: Comparison Between Paralle Back Squats and Belt Squats. J Human Kinetics. 2020; 72: 223-228. PubMed: https://pubmed.ncbi.nlm.nih.gov/32269663/

7. Florimond V. Basics of Surface ELectromyography: Applied to Physical Rehabilitation \& Biomechanics. Thought Technology Ltd. 2010; 18-19.

8. Gullett J, Tillman M, Gutierrez G, Chow J. A biomechanical comparison of back and front squats in healthy trained individuals. J Strength Cond Res. 2009; 23: 284-292.

PubMed: https://pubmed.ncbi.nlm.nih.gov/19002072/

9. Basmajian J, Deluca C. Muscle Alive. Baltimore: Williams \& Wilkins. 1985

10. Pereira G, Leporace G, Das Virgens Chagas D, Furtado L, Praxedes J, et al. Influence of hip extrenal rotation on hip adductor \& rectus femoris myoelectric activity during a dynamic parallel squat. J Strength Cond Res. 2010; 24: 2749-2754.

PubMed: https://pubmed.ncbi.nlm.nih.gov/20651607/

11. Escamilla R, Fleisig G, Zheng N, Lander J, Barrentine S, et al. Effects of technique variations on knee biomechanics during the squat and leg press. Med Sci Sport Exer. 2001; 33: 1552-1566. PubMed: https:// pubmed.ncbi.nlm.nih.gov/11528346/

12. Schwanbeck S, Chilibeck $P$, Binsted $G$. A comparison of free weight squat to Smith machine squat using electromyography. J Strength Cond Res. 2009; 23: 2588-2591.

PubMed: https://pubmed.ncbi.nlm.nih.gov/19855308/

13. Wilk K, Escamilla R, Fleisig G, Barrentine S, Andrews J, et al. A comparison of tibiofemoral joint ofrces and electromyographic activity during open and closed kinetic chain exercises. Am J Sports Med. 1996; 24: 518-527.

PubMed: https://pubmed.ncbi.nlm.nih.gov/8827313/
14. Bolgla L, Malone T, Umberger B, Uhl T. Hip strength and hip knee kinematics during stair descent in females with and without patellofemoral pain syndrome. J Orthopedic Sports Physical Therapy. 2008; 38: 12-18.

PubMed: https://pubmed.ncbi.nlm.nih.gov/18349475/

15. Fauth MG, Lutsch B, Gray A, Szalkowski C, Wurm B, et al. Hamstrings, quadriceps, \& gluteal muscle activation during resistance training exercises, in: ISBS Conference. 2010.

16. Myer G, Chu D, Brent J, Hewett T. Trunk \& hip control neuromuscular training for prevention of knee injury. Clin Sports Med. 2008; 27: 425-448. PubMed: https://pubmed.ncbi.nlm.nih.gov/18503876/

17. Piva S, Goodnite E, Childs J. Strength around the hip and flexibility of soft tissue in individuals with and without patellofemoral pain syndrome. J Orthop Sports Phys Ther. 2005; 35: 793-801.

PubMed: https://pubmed.ncbi.nlm.nih.gov/16848100/

18. Prins $M$, Van der Wurff $P$. Females with patellofemoral pain syndrome have weak hip muscles: A systematic review. Aust J Physiother. 2009, 55: 9-15.

PubMed: https://pubmed.ncbi.nlm.nih.gov/19226237/

19. Robinson R, Nee R. Analysis of hip strength in females seeking physical therapy treatment for unilateral patellofemoral pain syndrome. J Orthop Sports Phys Ther. 2007; 37: 232-238.

PubMed: https://pubmed.ncbi.nlm.nih.gov/17549951/

20. Khayambashi K, Ghoddosi N, Straub R. Hip Muscle Strength Predicts Noncontact Anterior Cruciate Ligament Injury in Male and Female Athletes: A Prospective Study. Am J Sports Med. 2015; 44: 355-361. PubMed: https://pubmed.ncbi.nlm.nih.gov/26646514/

21. Beardsley C. How important are the hamstrings during squats? Strength \& Conditioning Research, 2013.

22. Ebben W, Long N, Pawlowski Z, Chimielewski L, Clewien R, et al. Using squat repetition maximum testing to determine hamstring resistance training exercise loads. J Strength Cond Res. 2010; 24: 293-299. PubMed: https://pubmed.ncbi.nlm.nih.gov/20072071/

23. Hung Y, Gross M. Effect of Foot Position on Electromyographic Activity of the Vastus Medialis Oblique and Vastus Lateralis During LowerExtremity Weight-Bearing Activities. J Orthop Sports Phys Ther. 1999; 29: 93-105.

PubMed: https://pubmed.ncbi.nlm.nih.gov/10322584/

24. Murray N, Cipriani D, O'Rand D, Reed-Jones R. Effects of Foot Position during Squatting on the Quadriceps Femoris: An Electromyographic Study. Int J Exercise Sci. 2013; 6: 114-125. PubMed: https://pubmed.ncbi.nlm.nih.gov/27293497/ 